\title{
Remodelling of the bone marrow microenvironment by stromal hyaluronan modulates the malignancy of breast cancer cells
}

Xiaoyan Chen ${ }^{1,2+}$, Xiaoxing Shi ${ }^{3+}$, Yiwen Liu ${ }^{1}$, Yiqing He ${ }^{1}$, Yan Du ${ }^{1}$, Guoliang Zhang ${ }^{1}$, Cuixia Yang ${ }^{1,4^{*}}$ and Feng Gao ${ }^{1,4^{*}}$

\begin{abstract}
Background: Hyaluronan (HA) is an abundant component of the bone marrow (BM) extracellular matrix. Here, we investigated the abnormal deposition of HA in the BM microenvironment and its remodelling in mediating the malignancy of breast cancer cells (BCCs).

Methods: BCCs were transplanted into nude mice by intracardiac injection. The BCCs were cocultured with BMderived stromal HS5 cells. Then, the abnormal metabolism of HA and its correlation with the malignant growth and the intracellular signalling pathways of the BCCs were investigated. After knockdown/out of the HA receptor CD44 in cancer cells by shRNA and CRISPR/Cas9, the mechanism was investigated in vivo through intratibial inoculation and in vitro by coculture with HS5 cells.

Results: The malignancy of cancer cells was highly related to the degree of accumulation of HA in the BM. Further, stromal cell-derived HA, especially the mixed complex, significantly promoted the growth of BCCs and osteolysis by binding to the CD44 receptor. Additionally, the investigation of the underlying mechanism revealed that the PI3K, Cyclin D1, and CDK4 pathways were involved in the effect of bone stromal cell-derived HA on the BCC activities.
\end{abstract}

Conclusion: These data suggested that HA in abnormal BM stroma might be a therapeutic candidate for bone metastasis of breast cancer.

Keywords: Hyaluronan, Mammary tumour, Bone metastasis, Bone marrow stromal cells

\section{Background}

Malignant tumours are fatal diseases attributed to metastasis [1-3]. In breast cancer patients, bone is one of the most common sites for the localization of breast tumour cells $[4,5]$. Breast cancer is incurable once bone metastases occur, and this condition often severely affect

\footnotetext{
*Correspondence: gao3507@126.com; dr.steven@163.com

${ }^{+}$Xiaoyan Chen and Xiaoxing Shi contributed equally to this work.

'Department of Molecular Biology, Shanghai Jiao Tong University Affiliated Sixth People's Hospital, Shanghai 200233, People's Republic of China Full list of author information is available at the end of the article
}

the patient's quality of life [6]. Therefore, exploring the molecular mechanism of breast cancer distant metastasis and identifying new therapeutic target sites have become a research hotspot.

The bone marrow (BM) microenvironment contains many cytokines and high levels of extracellular matrix (ECM), which constitute the complex microenvironment of the BM. Hyaluronan (HA) is a nonprotein molecule in the BM matrix [7] and is one of the most abundant components of the ECM. The aberrant metabolism of HA plays an important role in tumorigenesis and

(c) The Author(s). 2020 Open Access This article is licensed under a Creative Commons Attribution 4.0 International License, which permits use, sharing, adaptation, distribution and reproduction in any medium or format, as long as you give appropriate credit to the original author(s) and the source, provide a link to the Creative Commons licence, and indicate if changes were made. The images or other third party material in this article are included in the article's Creative Commons licence, unless indicated otherwise in a credit line to the material. If material is not included in the article's Creative Commons licence and your intended use is not permitted by statutory regulation or exceeds the permitted use, you will need to obtain permission directly from the copyright holder. To view a copy of this licence, visit http://creativecommons.org/licenses/by/4.0/. The Creative Commons Public Domain Dedication waiver (http://creativecommons.org/publicdomain/zero/1.0/) applies to the data made available in this article, unless otherwise stated in a credit line to the data. 
development. For example, in the development of acute myeloid leukaemia, microenvironment remodelling resulting from the retention of $\mathrm{HA}$ in the $\mathrm{BM}$ matrix was significantly higher than that of normal $B M$ matrix [8]. However, few studies have focused on how HA alters the tumour-associated $\mathrm{BM}$ microenvironment. In humans, HA can be produced by many cell types, and stromal cells are believed to be the predominant source of HA [9]. Whether HA from BM stroma is altered and its subsequent effects on the growth of metastatic breast cancer cells (BCCs) in bone need to be elucidated.

In this study, we utilized in vivo models to study the remodelling of $\mathrm{HA}$ in tumour-associated BM stroma. The abnormal metabolism of $\mathrm{HA}$ and its correlation with the malignant growth of BCCs and the modifications of intracellular signalling pathways were investigated by in vitro coculture of BM stromal cells with BCCs. After knockout of the HA receptor CD44 in $\mathrm{BCCs}$, the receptor pathway of stromal HA on BCCs was investigated in vivo through intratibial inoculation and in vitro by coculture with HS5 cells.

\section{Materials and methods}

\section{Animal experiment}

Mice were obtained from Shanghai SLAC Laboratory Animal Co., Ltd. (China). All experimental protocols were approved by the appropriate institutional review board. Mice were subjected to tumour BM metastasis as previously described [10]. To model BM metastasis in mice, we delivered $7 \times 10^{5} \mathrm{BCCs}$ in $100 \mu \mathrm{L}$ of PBS via intracardiac injection into the left ventricle of 4- to 6week-old female nude mice. Nude mice aged 7-8 weeks were used for the intratibial experiments. For intratibial injection, the protocol was performed as previously described [11]. BCCs $\left(3 \times 10^{5}\right)$ in $10 \mu \mathrm{L}$ of PBS were injected into the tibias of the mice. The progression of bone destruction and the development of metastasis were monitored by micro-CT (Bruker, Germany).

\section{Cell lines}

The metastatic bone-seeking human breast cancer cell line MDA-MB-231BO was a kind gift from Prof. Shen (Department of Oncology, Shanghai Jiao Tong University Affiliated Sixth People's Hospital, Shanghai, China). The human BM stromal cell line HS5 and the highly malignant breast cancer cell line HS578T were obtained from the American Tissue Culture Collection (ATCC; USA). Cells were cultured at $37^{\circ} \mathrm{C}$ in a humidified atmosphere in the presence of $5 \% \mathrm{CO}_{2}$ and $95 \%$ air. MDA-MB-231BO and HS5 cell lines were cultured in high-glucose Dulbecco's modified Eagle's medium (DMEM; Gibco, USA) supplemented with 10\% foetal bovine serum (FBS; Gibco), 100 units $/ \mathrm{mL}$ penicillin (HyClone, USA), and $100 \mu \mathrm{g} / \mathrm{mL}$ streptomycin (HyClone). HS578T cells were maintained in high-glucose DMEM supplemented with $0.01 \mathrm{mg} / \mathrm{mL}$ human recombinant insulin, $10 \% \mathrm{FBS}, 100 \mathrm{units} / \mathrm{mL}$ penicillin, and $100 \mu \mathrm{g} / \mathrm{mL}$ streptomycin. MDA-MB-231BO and HS578T cells were infected with GFP fluorescent protein. Lentiviral particles were purchased from Hanbio Company (China). The desired number of BCCs was plated in complete culture medium and infected with lentiviral supernatant. Stable clones expressing GFP were selected via puromycin dihydrochloride (Santa Cruz Biotechnology, USA).

\section{Immunofluorescence staining}

HS5 cells were washed with PBS and fixed in cold methanol. After being fixed for $10 \mathrm{~min}$ at room temperature, the cells were blocked with $1 \%$ bovine serum albumin (BSA; Biofroxx, Germany) for $1 \mathrm{~h}$ and then stained with biotinylated a hyaluronan binding protein(HABP)antibody (Merck Millipore, Germany) at $4{ }^{\circ} \mathrm{C}$ overnight. Then, Alexa Fluor 488-conjugated streptavidin (Thermo Fisher Scientific, USA) and 4,6-diamidino2-phenylindole (DAPI) were used to stain the cells. The hyaluronidase(HAase, Sigma-Aldrich, USA)-treated group was used as the control. The cells were imaged using a fluorescence microscope (Nikon, Japan).

\section{Particle exclusion assay}

HS5 cells were cultured for $48 \mathrm{~h}$ in 48 -well plates. Aldolized erythrocyte cells (BD, USA) were added to the medium and allowed to settle for $15 \mathrm{~min}$ at room temperature. The cells were viewed by phase-contrast microscopy. The pericellular HA matrix was evidenced by halos surrounding the cells from which the fixed red blood cells were excluded. Finally, the cells were treated with HAase for $5 \mathrm{~min}$ and imaged.

\section{Determination of cell proliferation in the coculture system}

Coculture of cells was performed as previously described [12]. Briefly, HS5 BM stromal cells were seeded at $3 \times$ $10^{3}$ cells/per well in 96-well plates (Cellvis, USA) and incubated overnight. GFP-positive BCCs were seeded alone or layered on HS5 cells. The fluorescence intensity (FI) per well was read at $\lambda$ ex $488 \mathrm{~nm} / \lambda$ em $528 \mathrm{~nm}$ to detect the numbers of GFP-tumour cells with a Synergy 4 multidetection microplate reader (BioTek, USA) at day 0 and day 3 . The proliferation index was calculated by dividing FI in the same well at day 3/day 0 after subtracting the average of the background values of the corresponding condition (DMEM for GFP cultured alone or HS5 for cocultures).

\section{Western blot analysis}

BCCs were sorted by flow cytometry. Western blot analysis was performed as previously described [13]. The 
efficiency of gene interference and the changes in cellular signalling molecules after CD44 gene interference were also tested by western blot analysis. Cells were lysed on ice with complete RIPA buffer mixture (Beyotime, China) composed of protease inhibitor and phosphatase inhibitor and then lysed for $45 \mathrm{~min}$. The liquids were centrifuged at $15000 \mathrm{~g}$ for $10 \mathrm{~min}$, and the supernatant was boiled with loading sample buffer $(5 \times$, Beyotime) for $5 \mathrm{~min}$ at $100^{\circ} \mathrm{C}$. Proteins from each group were separated by SDS-PAGE and transferred to PVDF membranes (Merck Millipore). Blocking was carried out using $5 \%$ milk in TBST buffer at room temperature for $1 \mathrm{~h}$. The membranes were incubated at $4{ }^{\circ} \mathrm{C}$ overnight. The supplier and the specifications of each primary antibody were as follows: rabbit anti-PI3K polyclonal antibody, rabbit anti-CDK4 monoclonal antibody, rabbit anti-cyclin D1 monoclonal antibody, mouse anti-CD44 monoclonal antibody (Cell Signaling Technology, USA), and rabbit anti-HAS2 monoclonal antibody (Abcam, UK). We used GAPDH as an internal control for all analyses. The membranes were rinsed with TBST buffer three times. Subsequently, the membranes were incubated with HRP-conjugated anti-rabbit IgG (Liankebio, China) or HRP-conjugated anti-mouse IgG secondary antibodies (Liankebio) at room temperature for $1 \mathrm{~h}$ and washed three times with TBST. Immunoblotting was visualized using enhanced chemiluminescence solution (Merck Millipore), and a ChemiDocTM MP imaging system (BioRad, USA) was used to detect protein expression. Bands were quantified using ImageJ software.

\section{HA and growth factor detection}

The method was based on that of a previous report [14]. Before examination, the culture medium was centrifuged at $8000 \mathrm{~g}$ for $10 \mathrm{~min}$. The HA levels were determined by CLIA (New Industries Biomedical Engineering, China). The total protein content was measured using a BCA Protein Assay Kit (Thermo Fisher Scientific). The HA concentrations in the cell culture medium were routinely normalized to the total protein content of the cell after $72 \mathrm{~h}$ of culture and are expressed as nanogram (ng)/ microgram $(\mu \mathrm{g})$ total protein per $72 \mathrm{~h}$. HA extraction was performed as previously described [15]. The BM of the mouse tibia was immediately collected after sacrificing the animals. After digestion with proteinase $\mathrm{K}$, DNase and RNase (Sigma-Aldrich), the HA pellet was dissolved in $500 \mu \mathrm{L}$ of distilled water and incubated at $4{ }^{\circ} \mathrm{C}$. Aliquots were treated with $20 \mathrm{mg} / \mathrm{mL}$ HAase, incubated overnight at $37{ }^{\circ} \mathrm{C}$, and boiled at $100^{\circ} \mathrm{C}$. Growth factors, including TGF $\beta 1$, PDGF-BB, EGF, and IGF, were assayed by ELISAs according to the manufacturer's instructions (R\&D, USA).

\section{HA analysis by gel electrophoresis}

Twenty-five microlitres of each sample was mixed with $5 \mu \mathrm{L}$ of DNA loading solution (Thermo Fisher Scientific) and loaded onto a 1\% agarose gel (Amresco, USA). Ten microlitres of HA molecular size markers, which included markers at $15 \mathrm{MD}, 200 \mathrm{kD}$ and $10 \mathrm{kD}$, respectively, was run to determine the size of $\mathrm{HA}$ from each sample. The gel was placed in a $0.005 \%(w / v)$ Stains-All (Sigma-Aldrich) in 50\% ethanol solution overnight. For destaining, the gel was placed in distilled water for $24 \mathrm{~h}$ in the dark, placed under natural light in distilled water for $1 \mathrm{~h}$ to complete the final destaining stages and then photographed under white light.

\section{Cell growth assay}

Cell growth was determined using the Cell Counting Kit-8 (CCK-8, Dojindo, China) assay. Briefly, cells were seeded into each well of a 96-well plate and incubated at $5 \% \mathrm{CO}_{2}$ and $37{ }^{\circ} \mathrm{C}$ for $72 \mathrm{~h}$. Ten microlitres of CCK- 8 reagent was added to each well at the endpoint, and the plates were incubated for an additional $2 \mathrm{~h}$ at $37^{\circ} \mathrm{C}$. Finally, the absorbance of the control and experimental groups at $450 \mathrm{~nm}$ was measured with a spectrophotometer (Bio-Rad).

\section{Quantitative real-time PCR (qRT-PCR)}

Total RNA from cultured cells was extracted using TRIzol according to the manufacturer's instructions. The RNA quality and quantity were measured using a NanoDrop 2000 spectrophotometer (Thermo Fisher Scientific). For analysis of the expression of hyaluronan synthases (HAS), RNA samples were reverse transcribed using the PrimeScript $^{\text {tax }}$ RT reagent Kit (TaKaRa, China), and the relative mRNA expression levels were determined by qRT-PCR using TB Green Premix Ex Taq II (TaKaRa). GAPDH was used as an internal control. Primer sequences were as follows: HAS1, GGAATAACCTCTTGCAGCAG TTTC/GCCGGTCATCCCCAAAAG; HAS2, TCGCAAC ACGTAACGCAAT/ACTTCTCTTTTTCCACCCCATTT; HAS3, AACAAGTACGACTCATGGATTTCC/AGGCCAATGAAGTTCACCAC; GAPDH, CCCATGTTCG TATGGGTGT/TGGTCATGATCCTTCCACGATA. All qRT-PCR reactions were performed in a 7500 Real-Time PCR system (Applied Biosystems, Thermo Fisher Scientific).

\section{RNAi}

Double-stranded siRNA targeting HAS2 and control siRNA (catalogue number: siN05815122147) were designed and synthesized by RiboBio Co. (China). The corresponding target mRNA sequences for the siRNA were as follows:

si-HAS2, GTATCAGTTTGGTTT. HS5 cells $(3.5 \times$ $10^{5}$ ) were plated in 6-well plates. Twenty-four hours 
later, the HS5 cells were transfected with siRNA according to the manufacturer's protocol when cells reached $50 \%$ confluence. Briefly, $5 \mu \mathrm{L}$ of HAS 2 siRNA $(20 \mu \mathrm{mol} /$ L) was diluted in $150 \mu \mathrm{L}$ of Opti-MEM reduced serum medium (Opti-MEM, Thermo Fisher Scientific) and mixed with $9 \mu \mathrm{L}$ of Lipofectamine RNAiMAX transfection reagent (Thermo Fisher Scientific) prediluted in $150 \mu \mathrm{L}$ of Opti-MEM. After a 20 min incubation at room temperature, the complexes were added to the cells. Cells were transfected for $48 \mathrm{~h}$. HS5 cells transfected with a nontarget control siRNA were used as controls. The RNAi results were evaluated by qPCR and western blots.

\section{CD44 gene interference}

Oligonucleotides encoding short hairpin RNAs (shRNA) that target standard exons of CD44 were cloned into the plasmid vector Plkd-cmv-GFP-hU6 Puro (OBio, China). The plasmids were transfected into BCCs, and the cells were cultured for 2 weeks in the presence of puromycin $(4 \mu \mathrm{g} / \mathrm{mL}$; Sigma-Aldrich). BCCs transfected with Plkdcmv-GFP-hU6-control shRNA were used as controls. The shRNA results were evaluated by western blots.

The CD44 CRISPR/Cas9 KO plasmid, HDR plasmid and control CRISPR/Cas9 plasmid were purchased from Santa Cruz Biotechnology. BCCs $\left(5 \times 10^{4}\right)$ were plated on 24-well plates. After $24 \mathrm{~h}$ of incubation, the cells were transfected with CRISPR/Cas9 plasmids according to the manufacturer's protocol when they reached $60 \%$ confluence. The ratio of the target plasmid to the transfection reagent was $0.5 \mu \mathrm{g} / 2.5 \mu \mathrm{L}$. After $25 \mathrm{~min}$ of incubation at room temperature, the complexes were added to the cells. After the cells were expanded, cells with positive expression were sorted, and the effects of CD44 knockout were evaluated by western blots.

\section{Immunohistochemistry (IHC)}

For histological analyses of induced bone destruction lesions, IHC was performed on decalcified and paraffin sections. Tibial bones of nude mice were excised and fixed in $10 \%$ neutral-buffered formalin. The bone was decalcified, embedded in paraffin, sectioned, and stained. The expression of HA was indicated by detecting HABP in bone sections. Histological sections underwent 3amino-9-ethyl-carbazole (AEC, Dako, Denmark) IHC staining for HABP. HA expression was evaluated using ImageJ software.

\section{Statistical analysis}

Statistical analysis and image production were performed using GraphPad Prism 6.0 and Adobe Illustrator CC 2018. Data are expressed as the mean \pm SEM. Unpaired Student's $t$ test was used to compare two samples, and $p<0.05$ was considered statistically significant. Survival analysis was performed between two groups to compare the differences. The occurrence of bone destruction was set to " 1 ", and boneless destruction was set to "0". At different time points of scanning, the number of animals with bone destruction was indicated by the number " 1 ", and the number of animals without bone destruction was indicated by the number " 0 ". Group comparisons were performed using the Mantel-Cox test. When $p<0.05$, there was a significant difference between the two groups.

\section{Results \\ Abnormal metabolism of HA in the BM stromal microenvironment}

To explore whether HA in tumour-metastasized BM was abnormally metabolized, we constructed a model of breast cancer bone metastasis by injecting MDA-MB$231 \mathrm{BO}$ cells into the left ventricle of the mice. Osteolytic areas were confirmed by micro-CT scanning of the tibias of mice. There was obvious bone destruction in the BCC-injected group compared with the control group at 3 and 4 weeks (Fig. 1a). Normally, HA indicated by HABP staining was mainly distributed in the BM stroma (Fig. 1b). In experimental mice, the accumulation of HA in the BM stroma adjacent to bone was obviously increased after injection of MDA-MB-231BO cells into the left ventricle for 4 weeks (Fig. 1b). HA from the BM was analysed by gel electrophoresis. Notably, the HA level was abnormally increased in the MDA-MB-231BOtreated group compared with the control group (Fig. 1c).

Bone marrow HS5 stromal cells promoted BCC growth by enhancing proliferative signalling pathways

To verify the effects of the abnormal deposition of $\mathrm{HA}$ in the tumour-associated BM stroma on BCCs, we cocultured HS5 cells with high expression of HA (Fig. S1A and B) with BCCs in vitro.

BCCs infected with GFP fluorescent protein did not affect the growth of the cells themselves, as shown in Fig. 2a. In Fig. 2b, the results showed that HS5 cells could promote the growth of BCCs at either a low ratio (MDA-MB-231BO:HS5) of 1:2, a ratio of $1: 4$ or a high ratio of $1: 8$ at $24 \mathrm{~h}, 48 \mathrm{~h}$, and $72 \mathrm{~h}$, respectively. This promotion was particularly evident in the ratio of BCCs to stromal cells at 1:8 for 3 days of coculture. Figure $2 \mathrm{c}$ indicates that the number of MDA-MB-231BO-GFP cells was increased in the microenvironment matrix composed of HS5. To further investigate the downstream signalling pathways related to the promotion, we performed western blotting to detect PI3K, Cyclin D1, and CDK4 expression. After coculture with HS5 cells for 3 days, the expression levels of the PI3K, Cyclin D1, and CDK4 proteins were significantly increased in the MDAMB-231BO-GFP cells under HS5 coculture conditions (Fig. 2d). Taken together, these results showed that the HS5 cell matrix could promote the growth of MDA- 


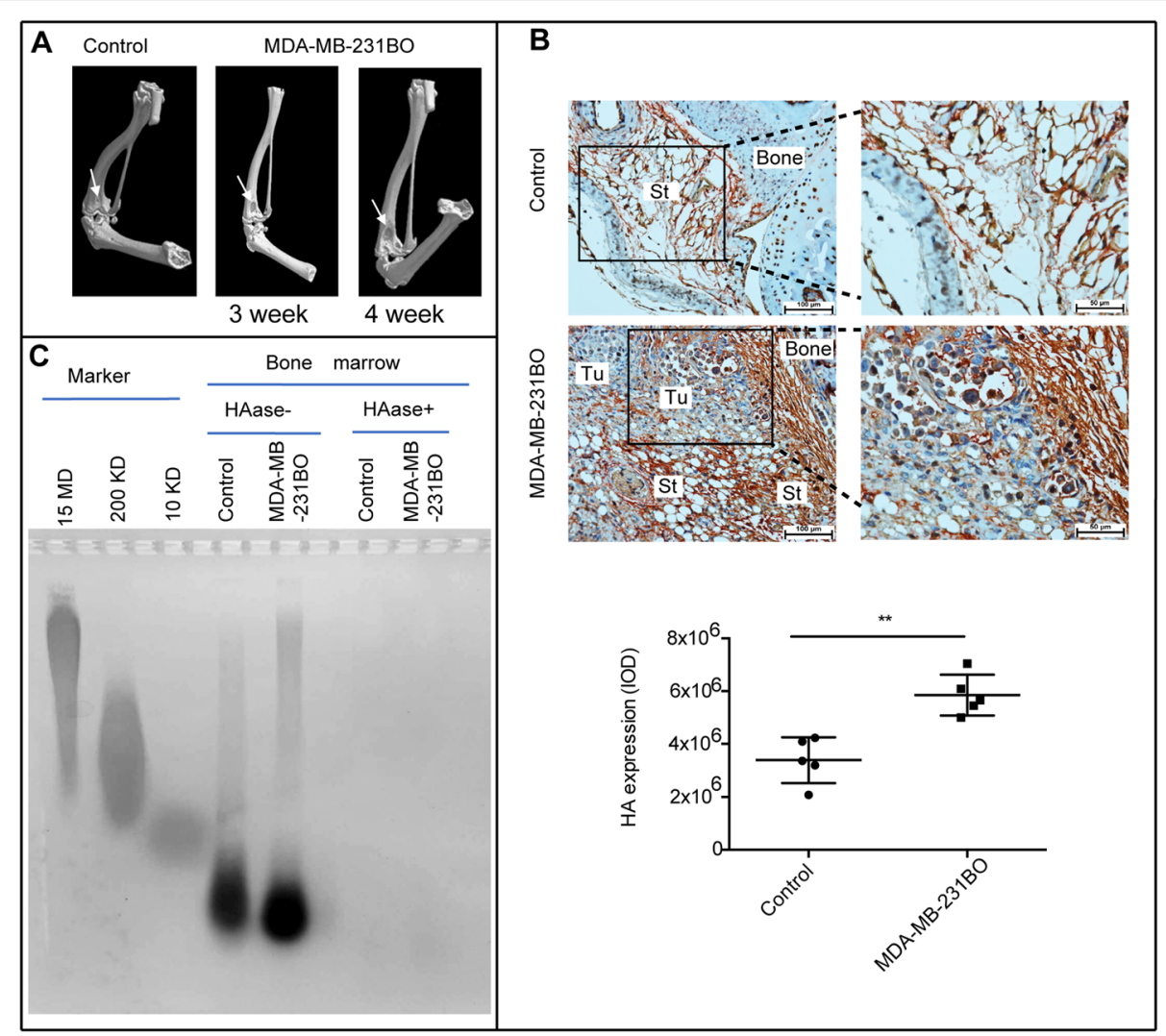

Fig. 1 Elevated deposition of HA in BM stroma. a. Micro-CT was used to scan the bone after MDA-MB-231BO cell intracardiac injection into the left ventricle of 4- to 6-week-old female nude mice. Arrows indicate the osteolytic areas in the control and treated groups. b.

Immunohistochemical expression of HA in BM. Brown indicates HA. Representative images of each group are shown. Left scale bars, $100 \mu$ m; right scale bars, 50 mm. Tu: tumour, St: stroma. Scatter plots showing the levels of HA. c. Elevated HA in breast cancer-associated BM stroma was analysed by electrophoresis

MB-231BO breast cancer cells by enhancing proliferative signal transduction. To further confirm these results, we performed the same experiment on an additional malignant breast cancer cell line, HS578T. First, HS578T cells were infected with GFP fluorescent protein and showed no changes in cell growth (Fig. S2A). After coculture with HS5 cells, the growth of the HS578T-GFP cells was promoted at either a low ratio (HS578T:HS5) of 1:2, a ratio of $1: 4$ or a high ratio of $1: 8$ at $24 \mathrm{~h}, 48 \mathrm{~h}$, and $72 \mathrm{~h}$, respectively (data are shown in Fig. S2B).

\section{HA mediated the growth of BCCs in the BM matrix microenvironment}

Next, we examined the remodelling of HA and its effects on the growth of BCCs. The culture supernatants of HS5 cells and MDA-MB-231BO cells were collected at $72 \mathrm{~h}$. The HA content was assayed as described previously (Fig. 3a). The results showed that compared to the BCCs, the HS5 stromal cells secreted more HA, suggesting that the HA in coculture was mainly derived from the HS5 stromal cells.
To explore whether the abnormal accumulation of HA could affect the growth of BCCs, we inhibited the synthesis of HA in coculture with an inhibitor of HA synthesis, 4-methylumpholone (4-MU), which inhibits HA synthesis mainly through two aspects: inhibition of the production of HAS, especially HAS2 and HAS3, at the post-transcriptional level [16, 17] and inhibition of the synthesis of UDP-GlcUA, which is a prerequisite for HA [16]. Different concentrations of 4-MU were applied to HS5 cells to determine the toxic effect, and the maximum working concentration of 4-MU, $300 \mu \mathrm{mol} / \mathrm{L}$ of 4MU (Fig. 3b), was selected, which had no toxic effects (Fig. 3c). After treatment with 4-MU, the HA content was significantly reduced (Fig. $3 \mathrm{~d}$ ), and the proliferation of BCCs was significantly attenuated (Fig. 3e). Our data indicated that the inhibition of $\mathrm{BCC}$ growth was mediated by HA within the culture context.

\section{Altered expression of HA in stromal cells affected the growth of BCCs}

HAS is the main functional enzyme of HA synthesis in cells and includes HAS1, HAS2, and HAS3 $[18,19]$. We 


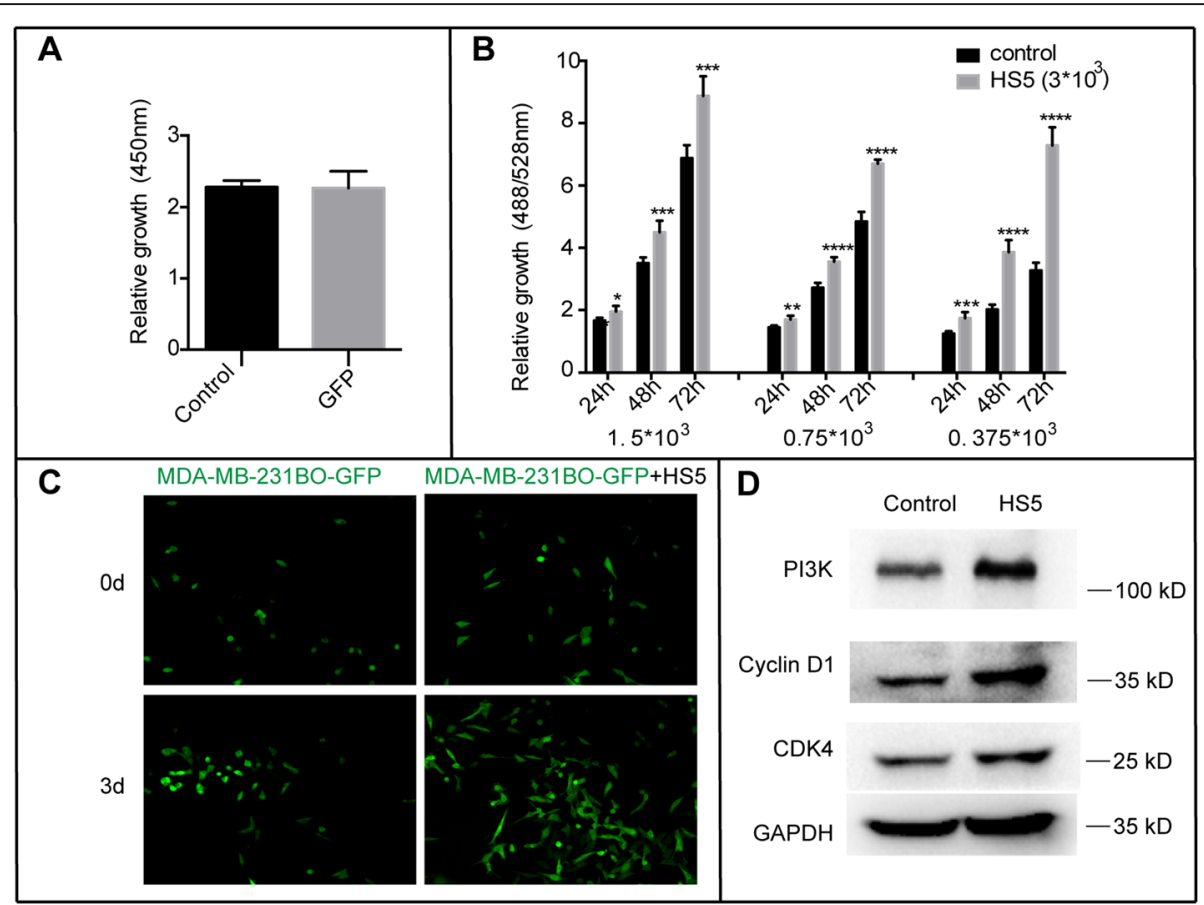

Fig. 2 The BM stromal cell line HS5 promoted MDA-MB-231BO-GFP cell proliferation. a. The effect of GFP protein on the growth of MDA-MB$231 \mathrm{BO}$ cells was analysed by CCK-8 assays. $\mathbf{b}$. The growth of MDA-MB-231BO-GFP cells after coculturing with HS5 stromal cells was evaluated by detecting the fluorescence intensity of GFP. The asterisk indicates that the relative growth of MDA-MB-231BO-GFP cells in the coculture environment was significantly promoted compared with that of the control group of MDA-MB-231BO-GFP cells alone. Bars represent the mean \pm SEM $\left({ }^{*} p<0.05,{ }^{* *} p<0.01,{ }^{* * *} p<0.005\right.$ and ${ }^{* * *} p<0.0001$ by unpaired Student's $t$ test). c. Fluorescence graphs show the number of MDA-MB231BO-GFP cells after coculture with stromal HS5 cells or culture alone for 0 and 3 days. d. After coculture with HS5 cells for three days, the proliferation-related proteins PI3K, Cyclin D1, and CDK4 in MDA-MB-231BO-GFP cells were detected by western blots

further explored whether BM stromal cell-derived HA promotes the proliferation of BCCs by decreasing the expression of HA in HS5 cells. We first found that the mRNA expression of HAS2 was significantly higher than that of HAS1 and HAS3, as shown in Fig. 4a. Next, we transfected siRNA targeting HAS2 and control siRNA into HS5 stromal cells. The data showed that HAS2 expression was significantly reduced by $85.16 \%$ at the mRNA level (Fig. 4b), following a significant parallel decrease in HAS2 protein expression (Fig. 4c). Figure 4d shows that HAS2 siRNA significantly decreased the amount of HA secreted by the cells but did not affect the growth of HS5 cells compared to control siRNA (Fig. 4e). Then, the HS5 cells were cocultured with BCCs after HAS2 siRNA interference. Figure $4 \mathrm{f}$ and Fig. S3A show that the control siRNA did not influence the effects of HS5 on the proliferation of MDA-MB-231BOGFP and HS578T-GFP cells. However, the growth of BCCs was significantly attenuated after coculture with HAS2 knockdown HS5 cells (Fig. 4g and Fig. S3B), suggesting that the expression of HA in stromal cells plays a role in mediating $\mathrm{BCC}$ proliferation. We also found that the proliferation-promoting effect of HS5 could not be completely eliminated after HAS2 siRNA interference. It is possible that HA synthesis could not be completely inhibited since HS5 contains synthases other than HAS2. Moreover, HS5 supernatant contains some active growth factors, such as TGF $\beta 1$, PDGF-BB, EGF, and IGF (Fig. S4), which may also contribute to growth.

\section{The effects of the HA receptor CD44 on the growth of MDA-MB-231BO cells in vitro and the progression of osteolytic lesions in bone}

CD44 is the major receptor of HA, and most of the activities of HA on cells are mediated through CD44 [20]. To investigate whether $\mathrm{CD} 44$ promoted $\mathrm{BCC}$ proliferation in a bone microenvironment, we knocked down the expression of CD44 in MDA-MB-231BO cells through shRNA lentivirus (Fig. 5a). Knockdown of CD44 did not affect the growth of MDA-MB-231BO cells (Fig. 5b and Fig. S5A) or related signalling pathways (Fig. S5B). As a result, in coculture experiments, HS5 stromal cells showed no effects on the proliferation of MDA-MB231BO-CD44 shRNA cells $(p<0.0001)$ (Fig. 5b). To further verify that the effect of HA on BCCs was through the CD44 signalling pathway, we knocked out CD44 by CRISPR (Fig. 5c) and injected the $\mathrm{CD} 44^{-/-}$cells into 




Fig. $3 \mathrm{HA}$ mediated the proliferation of MDA-MB-231BO-GFP cells in the matrix microenvironment. a. The HA content in the culture supernatant was assayed by CLIA. $\left(^{* * *} p<0.0001\right)$. b. The growth of HS5 cells was detected by CCK-8 assays after adding $100 \mu \mathrm{mol} / \mathrm{L}, 300 \mu \mathrm{mol} / \mathrm{L}, 500 \mu \mathrm{mol} / \mathrm{L}$, $700 \mu \mathrm{mol} / \mathrm{L}$, and $1000 \mu \mathrm{mol} / \mathrm{L} 4-\mathrm{MU}$ for 3 days. ${ }^{* *} p<0.01$ and ${ }^{* *} p<0.005$ compared with the control group as measured by unpaired Student's $t$ test). c. The growth of MDA-MB-231BO cells was detected by CCK-8 assays after adding 300 umol/L 4-MU for 3 days. d. HS5 cells were pretreated with $300 \mu \mathrm{mol} / \mathrm{L}$ 4-MU. Twenty-four hours later, MDA-MB-231BO-GFP cells were added. Furthermore, treatment with $300 \mu \mathrm{mol} / \mathrm{L}$ 4-MU was continued in the coculturing system for 3 days. The HA content in the supernatants was determined by CLIA. $\left({ }^{* * * *} p<0.0001\right.$ compared with the control group as measured by unpaired Student's $t$ test). e. The fluorescence intensity of GFP was used to measure the growth of MDA-MB231BO-GFP cells, which were separately treated with DMSO alone or cocultured with stromal HS5 cells before and after treatment with $300 \mu \mathrm{mol} /$ L 4-MU. ( ${ }^{*} p<0.05$ and ${ }^{* * *} p<0.0001$ compared with the control group as measured by unpaired Student's $t$ test). Bars represent the mean \pm SEM

mice through the tibia. Figure $5 \mathrm{~d}$ shows that the number of animals that underwent bone destruction in the MDA-MB-231BO ${ }^{\mathrm{CD} 44-/-}$ cell injection group was less than that in the control group. Figure $5 \mathrm{e}$ shows the micro-CT images of tibial destruction and demonstrates that the tibial destruction of the MDA-MB-231BO ${ }^{\mathrm{CD} 44}$ -I- cell injection group was weaker than that of the MDA-MB-231BO cell injection group. Supplementary Table lists the specific number of bone destructions in the two groups. The results further demonstrated that the remodelling of $\mathrm{HA}$ in the stroma may modulate metastatic BCC growth through the receptor CD44.

\section{Discussion}

The growth of tumour cells from primary lesions to micrometastases in distant organs is the leading cause of death in cancer patients [2], in which the microenvironment of metastatic organs plays a vital role [21]. For breast cancer, bone is the main metastatic site, and the BM microenvironment provides a supportive environment to regulate the progression of tumour cells [22]. $\mathrm{HA}$ is one of the most abundant ECM components enriched in the BM microenvironment [23]. Under normal conditions, the metabolism of ECM is physiologically constant, and the synthesis and degradation of HA are balanced. However, during the progression of pathological destruction, such as cancer, HA may be abnormally metabolized, remodelling the microenvironment of the ECM. For instance, in the development of acute myeloid leukaemia, the retention of $\mathrm{HA}$ in the $\mathrm{BM}$ matrix was significantly higher than that of normal BM matrix [8]. At present, it is still unknown how stromal $\mathrm{HA}$ is metabolized in tumour metastatic BM and to what extent HA remodelling modulates cancer cell malignancy. In this study, we first established a bone metastatic model through intracardiac injection with highly bone metastatic BCCs, MDA-MB-231BO cells. The results showed that stromal HA in the BM was significantly increased after injection, which was identical to the findings of other studies $[24,25]$. These reports showed that in mouse breast cancer xenograft models, HA accumulation in bone metastatic lesions was perturbed peripherally, and administration of either 4-MU or oligosaccharides of hyaluronan (oHA) decreased the expression of stromal HA and inhibited the expansion of osteolytic lesions $[24,25]$.

As there are no reports on the direct effects of bone stromal HA on breast cancer growth, we next developed an in vitro model to mimic the BM microenvironment for an HA remodelling study, in which a BM-derived 


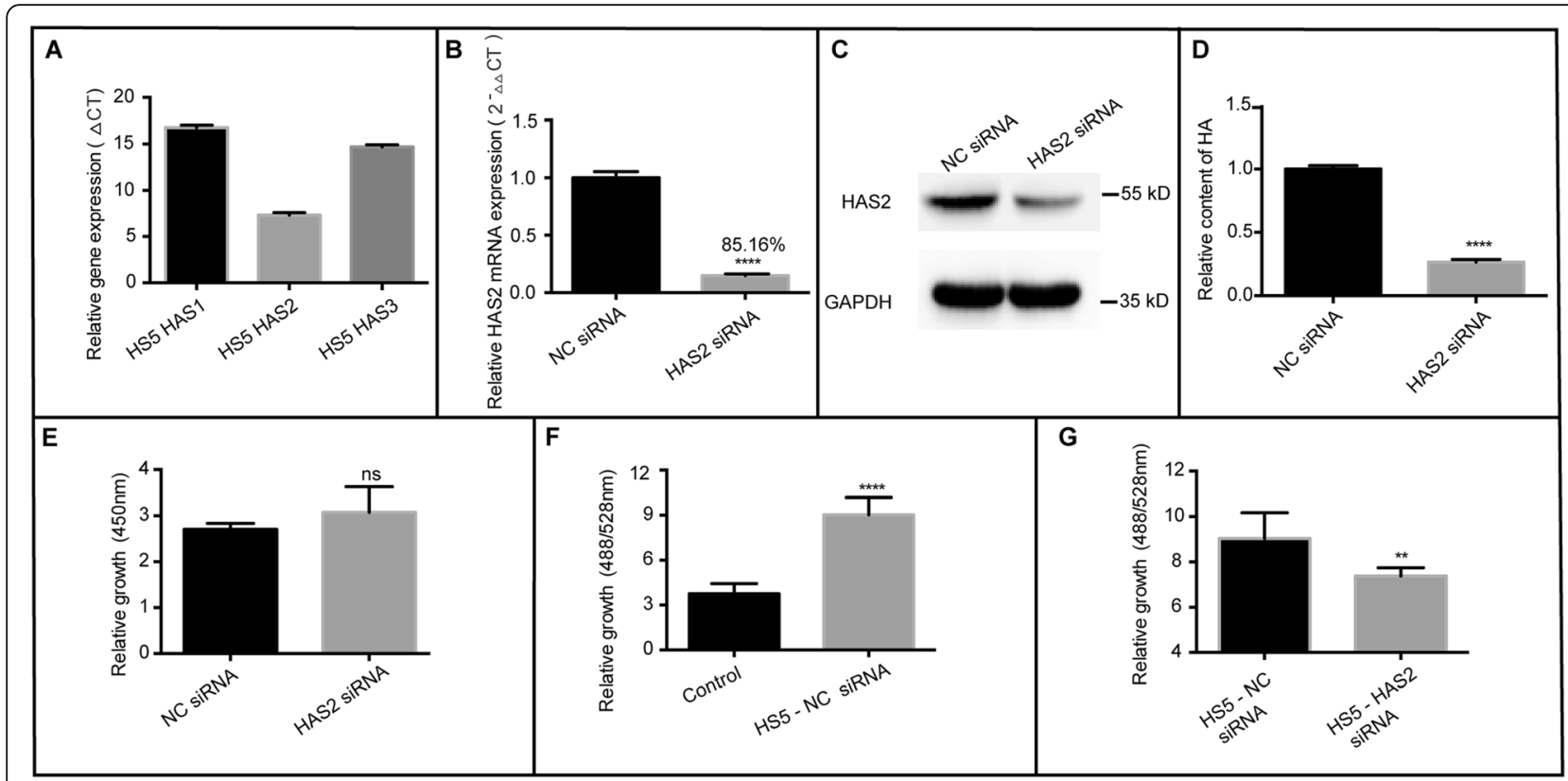

Fig. 4 HA derived from HS5 stromal cells affected the growth of MDA-MB-231BO-GFP cells. a. The expression of three HA synthases, HAS1, HAS2, and HAS3, in HS5 cells was detected by qPCR. $\mathbf{b}$. The interference efficiency of HAS2 siRNA was evaluated by qPCR in HS5 cells. c. The expression of HAS2 was detected by western blots after interference with HAS2 siRNA in HS5 cells. d. HA content in the supernatant was determined after HAS2 expression was downregulated in HS5 cells. ${ }^{* * * *} p<0.0001$ compared with the NC siRNA group as measured by unpaired Student's $t$ test). e. The growth of HS5 cells after downregulation of HAS2 was detected by CCK-8 assays. F. A total of 3000 HS5-NC siRNA cells/well were plated in special 96-well plates. Twenty-four hours later, $0.375 \times 10^{3}$ MDA-MB-231BO-GFP cells were plated in the HS5 cell wells. MDA-MB-231BO-GFP cells were cultured alone as controls. After three days of culture, the fluorescence intensity of GFP was determined (**** $p<0.0001$ compared with the control group as measured by unpaired Student's $t$ test). G. After HAS2 in HS5 cells was knocked down by HAS2 siRNA, the cells were cocultured with MDA-MB-231BO-GFP cells for $72 \mathrm{~h}$. The fluorescence intensity of GFP was measured. ${ }^{* *} p<0.01$ compared with the HS5-NC siRNA group as measured by unpaired Student's $t$ test). Bars represent the mean \pm SEM

stromal cell line, HS5, was selected for its high expression of HA. We found that HS5 cells could promote the growth of BCCs in coculture through the activation of PI3K signalling transduction, which is an important pathway in the proliferation of tumour cells [26]. Moreover, the cell cycle regulators cyclin D1 and CDK4, which promote cell cycle progression [27-29], were obviously elevated in the BCCs. These results indicated that HS5 provided an environment conducive to cancer cell proliferation.

Previous studies have indicated that in breast cancer bone metastasis, HA from cancer stem cells promoted the expression of PDGF-BB in tumour-associated macrophages, induced the activation of HS5 cells and promoted the growth of breast cancer stem-like cells [30]. In our experiments, the HA levels secreted by HS5 were significantly higher than those secreted by BCCs; therefore, we focused on whether HS5 stromal cell-derived $\mathrm{HA}$ is associated with breast cancer cell growth. When HA expression was inhibited by 4-MU, a widely recognized specific inhibitor of HA synthesis, in the coculture environment, the promotion of HS5 cells on BCCs was significantly decreased, implying that HA played an important role in promoting the proliferation of BCCs.
Importantly, when HAS2 was inhibited in HS5 cells, the secretion of HA was substantially reduced, and the proliferative ability of the BCCs was thus weakened. The results indicated that HA derived from HS5 cells played a key role in promoting the growth of BCCs. However, the reduction of $\mathrm{HA}$ in the coculture environment did not completely abolish the promotion of the proliferation of MDA-MB-231BO cells, suggesting that HA synthesis might not be completely inhibited after silencing of the HAS2 gene or HA may not be the only factor involved in the proliferative effect on BCCs. As we demonstrated in the supplements, some proliferation-promoting factors, such as TGF $\beta 1$, PDGF-BB, EGF, and IGF, are detectable in the culture supernatants of HS5 cells. These cytokines must be active in our coculture system. However, our present study focused on HS5-derived HA on BCC proliferation, which was confirmed by both 4-MU inhibition and HAS2 interference experiments. The results suggest the effects of stromal HA on cell growth but cannot exclude the effects of other cytokine activities. Further detailed studies are needed.

Moreover, the difference in molecular weight and tissue content can lead to differences in HA activity [31, 32]. It is now accepted that high molecular weight HA (HMW- 


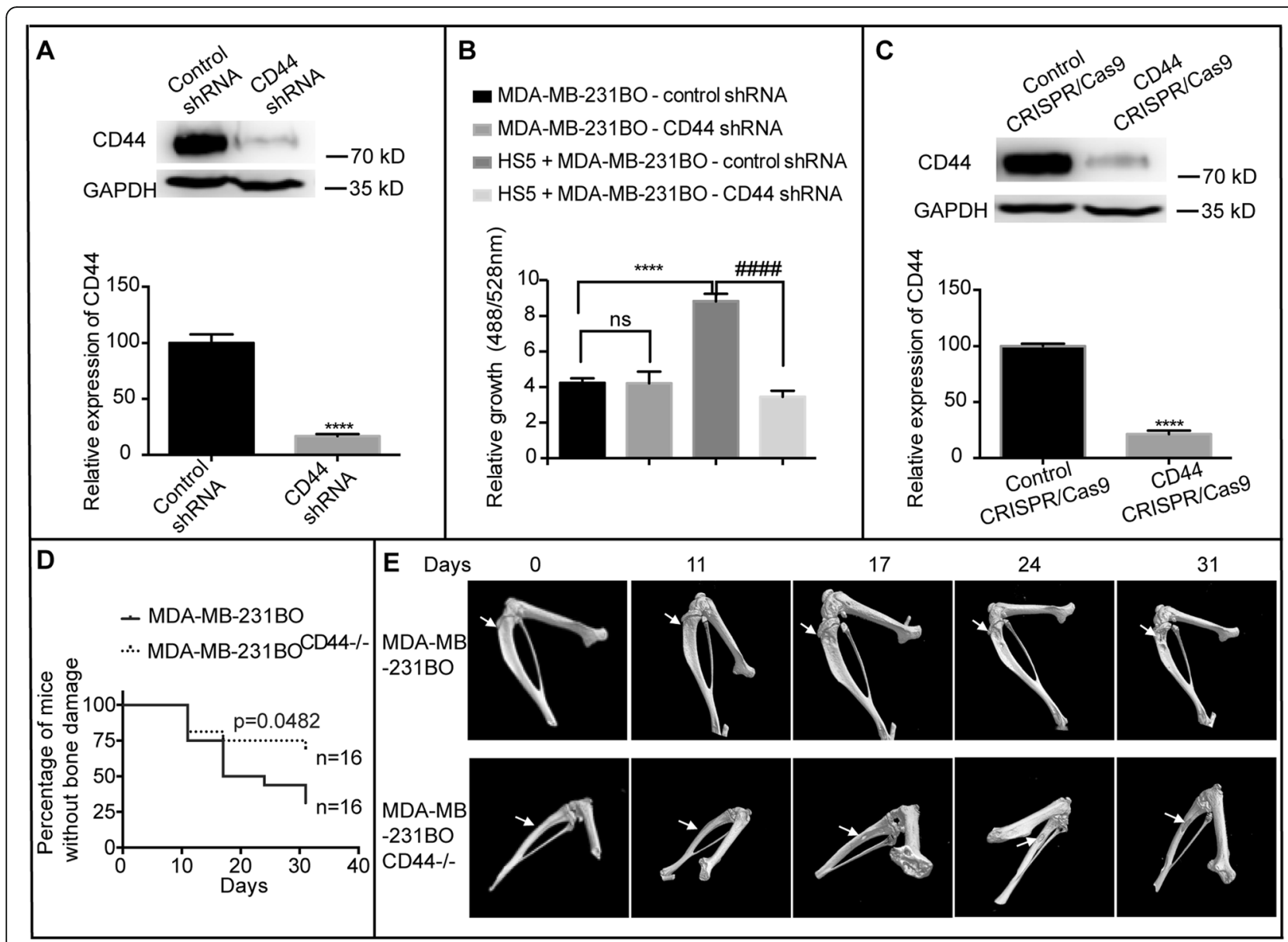

Fig. $5 \mathrm{HA}$ promoted the growth of MDA-MB-231BO cells via the CD44 receptor. a. The knockdown effect of CD44 shRNA on MDA-MB-231BO cells was detected by western blots. The band intensities were analysed by densitometry analysis $\left({ }^{* * *} p<0.0001\right.$ compared with the control shRNA group as measured by unpaired Student's $t$ test). b. MDA-MB-231BO-CD44 shRNA cells and MDA-MB-231BO-control shRNA cells were cocultured with HS5 cells for $72 \mathrm{~h}$. The fluorescence intensity of GFP was measured. Data are presented as the mean \pm SEM $\left({ }^{* * * *} p<0.0001\right.$ compared with the MDA-MB-231BO-control shRNA cells alone group as measured by unpaired Student's $t$ test, \#\#\#\# $p<0.0001$ compared with the group of HS5 cells cocultured with MDA-MB-231BO-control shRNA cells as measured by unpaired Student's $t$ test). c. The knockout efficiency of CD44 in MDA-MB-231BO cells was detected by western blots. The band intensities were analysed by densitometry analysis $\left({ }^{* * * *} p<0.0001\right.$ compared with the control CRISPR/Cas9 group as measured by unpaired Student's $t$ test). d. MDA-MB-231BOCD44-/- cells and MDA-MB-231BO cells were injected into the tibia of mice, and the tibias were monitored on days $0,11,17,24$ and 31. $n=16$ ( $p=0.0482$ compared with the MDAMB-231BO-treated group by the Mantel-Cox test). e. The micro-CT images showed the bone destruction of the two groups at days $0,11,17,24$, and 31 after the injection. Arrows indicate the site of bone damage

HA) usually inhibits cell proliferation [33], while low molecular weight HA (LMW-HA) may promote proinflammatory events [34, 35]. Our previous study also showed that simply concentrated HMW-HA can arrest breast cancer cell cycle progression, which seems to be contradicted by the present study [36]. Such diversity may be due to HA MW heteropolysaccharides and tissue distribution; for example, there are thousands of HA molecules of different sizes in tumour remodelling microenvironments. The present study on HS5-derived HA includes various fragments of HA that mostly mimic the in vivo microenvironments, therefore resulting in a promotion of cancer cells. Others have also shown that accumulation and increased levels of HA contribute to tumour progression [20].
Overall, it is worth further investigating the details of HA in cancer progression and metastasis, especially the abnormal metabolism of HA in tumour microenvironments.

Most of the functions of HA are mediated through binding to the cell receptor, and CD44 is the main receptor for HA [20]. The binding of HA to CD44 induces conformational changes to the receptor as well as post-translational modifications that regulate downstream signalling pathways, resulting in numerous pathobiological processes, including inflammation, wound healing, tumour growth, and metastasis [37-40]. In this study, CD44 was first knocked down in BCCs, and coculture experiments with HS5 cells were repeated as before. The results indicated that the HS5 cells showed no effects on BCC growth after the 
downregulation of CD44 expression. Because CD44 can influence the metastasis and invasion of cancer cells [41, 42], we next performed an in vivo experiment with CD44 knockout $\mathrm{BCCs}$ directly injected into the mouse through the tibia instead of the left ventricle. The results showed that the number of mice showing osteolytic bone metastasis and the extent of bone destruction in the $\mathrm{CD} 44^{-/-}$cell injection group were significantly decreased. The data suggested that the effects of HA-mediated bone microenvironment remodelling after $\mathrm{BCC}$ infiltration may be conducted through binding to CD44.

\section{Conclusions}

In summary, our results showed that stromal HA in tumourassociated BM was abnormally distributed and that remodelling of the microenvironment may influence the growth of BCCs. The effects of HA on cancer cells may be mediated through its receptor CD44, which triggers a cascade of signal transduction increases, including increases in PI3K, Cyclin D1 and CDK4. Notably, the abnormal deposition of HA was mainly derived from tumour-associated BM stroma. These findings suggest the potential of stromal HA in bone as a target for treating bone metastasis of breast cancer.

\section{Supplementary information}

Supplementary information accompanies this paper at https://doi.org/10. 1186/s12964-020-00592-z.

Additional file 1: Figure S1. The high expression of HA in HS5 cells. A. The expression of HA on HS5 cells was analysed by immunofluorescence. Green indicates HA and blue represents DAPI. B. HS5 cells were cultured for $48 \mathrm{~h}$. HA in pericellular matrix was visualized by particle exclusion. The results showed that HS5 cells highly expressed HA on the surface and formed pericellular HA matrix.

Additional file 2: Figure S2. BM stromal cell HS5 promoted HS578TGFP cells proliferation. A. The effect of GFP protein on the growth of HS578T cells was determined by CCK-8 assays. B. The growth of HS578TGFP cells after coculturing with stromal cell HS5 was evaluated by detecting the fluorescence intensity of GFP. The asterisk indicates that the relative growth of HS578T-GFP cells in the coculture environment was significantly promoted compared with that of the control group of HS578T-GFP alone. Bars represent mean \pm SEM. ( ${ }^{*} p<0.05,{ }^{* *} p<0.01$, ${ }^{* * *} p<0.005$ and ${ }^{* * * *} p<0.0001$ by unpaired Student's $t$ test).

Additional file 3: Figure S3. HA derived from stromal cell HS5 affected the growth of HS578T-GFP cells. A. A total of 3000 HS5-NC siRNA cells/ well were plated in special 96-well plates. Twenty-four hours later, $0.375 \times 10^{3}$ HS578T-GFP cells were plated in the HS5 cell wells. HS578TGFP cells were cultured alone as controls. After three days of culture, the fluorescence intensity of GFP was determined, ${ }^{* * * *} p<0.0001$ compared with control group as measured by unpaired Student's $t$ test). B. After HAS2 in HS5 cells was knocked down by HAS2 siRNA, the cells were cocultured with HS578T-GFP cells for $72 \mathrm{~h}$. The fluorescence intensity of GFP was measured. (*** $p<0.005$ compared with HS5-NC siRNA group as measured by unpaired Student's $t$ test). Bars represent mean \pm SEM.

Additional file 4: Figure S4. Levels of cytokines measured from HS5 culture supernatants. Various cytokines in the culture supernatants were measured by ELISAs.

Additional file 5: Figure S5. The effect of CD44 on breast cancer cell growth. A. CCK-8 proliferation assay was used to detect the growth of MDA-MB-231BO cells after downregulation of CD44. Bars represent mean \pm SEM. B. Western blot was used to detect the expression of signalling proteins, including PI3K, Cyclin D1, and CDK4 after downregulation of CD44.

Additional file 6: Table 1. The number of mice of osteolysis.

\section{Abbreviations}

BM: Bone marrow; HA: Hyaluronan; BCCs: Breast cancer cells;

ECM: Extracellular matrix; ATCC: American Tissue Culture Collection; DMEM: Dulbecco's modified Eagle's medium; FBS: Foetal bovine serum; Fl: Fluorescence intensity; HAase: Hyaluronidase; CCK-8: Cell Counting Kit-8; HAS: Hyaluronan synthase; shRNA: Short hairpin RNA;

IHC: Immunohistochemistry; AEC: 3-amino-9-ethyl-carbazole; MW: Molecular weight; OHA: Oligosaccharides of hyaluronan; 4-MU: 4-methylumpholone

\section{Acknowledgements}

Not applicable.

\section{Authors' contributions}

Xiaoyan Chen, Xiaoxing Shi, and Feng Gao designed and approved the submitted version. Xiaoyan Chen, Yiqing $\mathrm{He}$, and Yiwen Liu performed the research and collected data. Xiaoyan Chen, Xiaoxing Shi, Cuixia Yang, and Guoliang Zhang analysed the data. Xiaoyan Chen, Xiaoxing Shi, Yan Du, Cuixia Yang and Feng Gao wrote and revised the manuscript. All authors read and approved the final manuscript.

\section{Funding}

This work was supported by grants from the National Natural Science Foundation of China (81572821, 81672843, 81702852, and 81872357), the Shanghai Municipal Education Commission-Gaofeng Clinical Medicine Grant Support (20171924), the Program of Shanghai Leading Talents (2013-038), the Doctor Innovation Fund of Shanghai Jiao Tong University School of Medicine (BXJ201944) and the Shanghai Pujiang Program (2019PJD037).

\section{Availability of data and materials}

The datasets used and/or analysed during the current study are available from the corresponding author on reasonable request.

\section{Ethics approval and consent to participate}

All experimental protocols were approved by the appropriate institutional review board.

\section{Consent for publication}

Not applicable.

\section{Competing interests}

The authors declare no competing interests.

\section{Author details}

${ }^{1}$ Department of Molecular Biology, Shanghai Jiao Tong University Affiliated Sixth People's Hospital, Shanghai 200233, People's Republic of China. ${ }^{2}$ College of Medical Technology, Shanghai University of Medicine and Health Sciences, Shanghai 201318, People's Republic of China. ${ }^{3}$ Department of Laboratory Medicine, Shanghai Wujing General Hospital, Shanghai 201103, People's Republic of China. ${ }^{4}$ Department of Clinical Laboratory, Shanghai Jiao Tong University Affiliated Sixth People's Hospital, Shanghai 200233, People's Republic of China.

Received: 12 December 2019 Accepted: 7 May 2020

Published online: 09 June 2020

\section{References}

1. Torre LA, Bray FRL, Siegel RI J, Ferlay JJ, Lortet-Tieulent JA, Jemal A. Global cancer statistics, 2012. CA Cancer J Clin. 2015;65:87-108.

2. Suva LJ, Washam CRW, Nicholas Rw RJ, Griffin RJ. Bone metastasis: mechanisms and therapeutic opportunities. Nat Rev Endocrinol. 2011;7:20818.

3. Bray F, Ferlay J, Soerjomataram I, Siegel RL, Torre LA, Jemal A. Global cancer statistics 2018: GLOBOCAN estimates of incidence and mortality worldwide for 36 cancers in 185 countries. CA Cancer J Clin. 2018;68:394-424. 
4. Kennecke H, Yerushalmi Y, Woods R, Cheang MCU, Voduc D, Nielsen CHSO, et al. Metastatic behavior of breast cancer subtypes. J Clin Oncol. 2010;28: $3271-7$

5. Liede A, Jerzak KJ, Hernandez RK, Wade SW, Sun P, Narod SA. The incidence of bone metastasis after early-stage breast cancer in Canada. Breast Cancer Res Treat. 2016;156:587-95.

6. Aielli F, Ponzetti M, Rucci N. Bone Metastasis Pain, from the Bench to the Bedside. Int J Mol Sci. 2019; 20: pii: E280.

7. Nguyen N, Kumar A, Chacko S, Ouellette RJ, Ghosh A. Human hyaluronic acid synthase-1 promotes malignant transformation via epithelial-tomesenchymal transition, micronucleation and centrosome abnormalities. Cell Commun Signal. 2017;15:48.

8. Sundström G, Dahl IM, Hultdin M, Lundström B, Wahlin A, Engström-Laurent A. Bone marrow hyaluronan distribution in patients with acute myeloid leukemia. Med Oncol. 2005:22:71-8.

9. Toole BP. Hyaluronan: from extracellular glue to pericellular cue. Nat Rev Cancer. 2004:4:528-39.

10. Tulotta C, Groenewoud A, Snaar-Jagalska BE, Ottewell P. Animal models of breast Cancer bone metastasis. Methods Mol Biol. 1914:2019:309-30.

11. Kobayashi A, Okuda H, Xing F, Pandey PR, Watabe M, Hirota S, et al. Bone morphogenetic protein 7 in dormancy and metastasis of prostate cancer stem-like cells in bone. J Exp Med. 2011;208:2641-55.

12. Ramasamy K, Khatun H, Macpherson L, Caley MP, Sturge J, Mufti GJ, et al. Fluorescence-based experimental model to evaluate the concomitant effect of drugs on the tumour microenvironment and cancer cells. Br J Haematol. 2012;157:564-79.

13. Zhang G, Guo L, Yang C, Liu Y, He Y, Du Y, et al. A novel role of breast cancer-derived hyaluronan on inducement of M2-like tumor-associated macrophages formation. Oncoimmunology. 2016;5:e1172154.

14. Wu M, Cao M, He Y, Liu Y, Yang C, Du Y, et al. A novel role of low molecular weight hyaluronan in breast cancer metastasis. FASEB J. 2015;29: 1290-8.

15. Tian X, Azpurua JC, Hine CA, Vaidya AM, Myakishev-Rempel MJ, Ablaeva JZ, et al. High-molecular-mass hyaluronan mediates the cancer resistance of the naked mole rat. Nature. 2013;499:346-9.

16. Kakizaki I, Kojima K, Takagaki K, Endo M, Kannagi R, Ito M, et al. A novel mechanism for the inhibition of hyaluronan biosynthesis by 4methylumbelliferone. J Biol Chem. 2004;279:33281-9.

17. Goncharova V, Serobyan N, lizuka S, Schraufstatter I, de Ridder A, Povaliy T, et al. Hyaluronan expressed by the hematopoietic microenvironment is required for bone marrow hematopoiesis. J Biol Chem. 2012;287:25419-33.

18. Bernert B, Porsch $H_{1}$ Heldin P. Hyaluronan synthase 2 (HAS2) promotes breast cancer cell invasion by suppression of tissue metalloproteinase inhibitor 1 (TIMP-1). J Biol Chem. 2011;286:42349-59.

19. Karalis TT, Heldin P, Vynios DH, Neill T, Buraschi S, lozzo RV, et al. Tumorsuppressive functions of 4-MU on breast cancer cells of different ER status: regulation of hyaluronan/HAS2/CD44 and specific matrix effectors. Matrix Biol. 2019;78-79:118-38.

20. Karousou E, Misra S, Ghatak S, Dobra K, Gotte M, Vigetti D, et al. Roles and targeting of the HAS/hyaluronan/CD44 molecular system in cancer. Matrix Biol. 2017:59:3-22.

21. Ungefroren $H$, Sebens $S$, Seidl $D$, Lehnert $H$, Hass R. Interaction of tumor cells with the microenvironment. Cell Commun Signal. 2011;9:18.

22. Gilbey AM, Burnett D, Coleman RE, Holen I. The detection of circulating breast cancer cells in blood. J Clin Pathol. 2004;57:903-11.

23. Zöller M. CD44, Hyaluronan, the hematopoietic stem cell, and leukemiainitiating cells. Front Immunol. 2015;6:235.

24. Urakawa H, Nishida Y, Wasa J, Arai E, Zhuo L, Kimata K, et al. Inhibition of hyaluronan synthesis in breast cancer cells by 4-methylumbelliferone suppresses tumorigenicity in vitro and metastatic lesions of bone in vivo. Int J Cancer. 2012;130:454-66.

25. Urakawa H, Nishida Y, Knudson W, Knudson CB, Arai E, Kozawa E, et al Therapeutic potential of hyaluronan oligosaccharides for bone metastasis of breast cancer. J Orthop Res. 2012;30:662-72.

26. Bahrami A, Khazaei M, Shahidsales S, Hassanian SM, Hasanzadeh M, Maftouh $\mathrm{M}$, et al. The therapeutic potential of PI3K/Akt/mTOR inhibitors in breast Cancer: rational and Progress. J Cell Biochem. 2018;119:213-22.

27. Wang G, Gormley M, Qiao J, Zhao Q, Wang M, Di Sante G, et al. Cyclin D1mediated microRNA expression signature predicts breast cancer outcome. Theranostics. 2018;8:2251-63.
28. Filipits M, Dafni U, Gnant M, Polydoropoulou V, Hills M, Kiermaier A, et al. Association of p27 and Cyclin D1 expression and benefit from adjuvant Trastuzumab treatment in HER2-positive early breast Cancer: a TransHERA study. Clin Cancer Res. 2018;24:3079-86.

29. Robert M, Frenel JS, Bourbouloux E, Rigaud DB, Patsouris A, Augereau P, et al. An update on the clinical use of CDK4/6 inhibitors in breast Cancer. Drugs. 2018;78:1353-62

30. Okuda H, Kobayashi A, Xia B, Watabe M, Pai SK, Hirota S, et al. Hyaluronan synthase HAS2 promotes tumor progression in bone by stimulating the interaction of breast cancer stem-like cells with macrophages and stromal cells. Cancer Res. 2012;72:537-47.

31. Slevin M, Kumar S, Gaffney J. Angiogenic oligosaccharides of hyaluronan induce multiple signaling pathways affecting vascular endothelial cell mitogenic and wound healing responses. J Biol Chem. 2002;277:41046-59.

32. Heldin P, Basu K, Olofsson B, Porsch H, Kozlova I, Kahata K. Deregulation of hyaluronan synthesis, degradation and binding promotes breast cancer. J Biochem. 2013;154:395-408.

33. Seluanov A, Hine C, Azpurua J, Feigenson M, Bozzella M, Mao Z, et al. Hypersensitivity to contact inhibition provides a clue to cancer resistance of naked mole-rat. Proc Natl Acad Sci U S A. 2009;106:19352-7.

34. Chistyakov DV, Astakhova AA, Azbukina NV, Goriainov SV, Chistyakov W, Sergeeva MG. High and low molecular weight hyaluronic acid differentially influences Oxylipins synthesis in course of Neuroinflammation. Int J Mol Sci. 2019;20.

35. Vistejnova L, Safrankova B, Nesporova K, Slavkovsky R, Hermannova M, Hosek $\mathrm{P}$, et al. Low molecular weight hyaluronan mediated CD44 dependent induction of IL- 6 and chemokines in human dermal fibroblasts potentiates innate immune response. Cytokine. 2014;70:97-103.

36. Chen X, Du Y, Liu Y, He Y, Zhang G, Yang C, et al. Hyaluronan arrests human breast cancer cell growth by prolonging the G0/G1 phase of the cell cycle. Acta Biochim Biophys Sin Shanghai. 2018;50:1181-9.

37. Noble PW, Jiang D. Matrix regulation of lung injury, inflammation, and repair: the role of innate immunity. Proc Am Thorac Soc. 2006:3:401-4.

38. Heldin P, Karousou E, Bernert B, Porsch H, Nishitsuka K, Skandalis SS Importance of hyaluronan-CD44 interactions in inflammation and tumorigenesis. Connect Tissue Res. 2008;49:215-8.

39. Sironen RK, Tammi M, Tammi R, Auvinen PK, Anttila M, Kosma VM. Hyaluronan in human malignancies. Exp Cell Res. 2011;317:383-91.

40. Veiseh M, Kwon DH, Borowsky AD, Tolg C, Leong HS, Lewis JD, et al. Cellular heterogeneity profiling by hyaluronan probes reveals an invasive but slow-growing breast tumor subset. Proc Natl Acad Sci U S A. 2014;111: E1731-9.

41. Ouhtit A, Abdraboh ME, Hollenbach AD, Zayed H, Raj MHG. CD146, a novel target of CD44-signaling, suppresses breast tumor cell invasion. Cell Commun Signal. 2017;15:45.

42. Ouhtit A, Elmageed ZYA, Abdraboh ME, Lioe TF, Raj MHG. In vivo evidence for the role of CD44s in promoting breast cancer metastasis to the liver. Am J Pathol. 2007:171:2033-9.

\section{Publisher's Note}

Springer Nature remains neutral with regard to jurisdictional claims in published maps and institutional affiliations.

Ready to submit your research? Choose BMC and benefit from

- fast, convenient online submission

- thorough peer review by experienced researchers in your field

- rapid publication on acceptance

- support for research data, including large and complex data types

- gold Open Access which fosters wider collaboration and increased citations

- maximum visibility for your research: over $100 \mathrm{M}$ website views per year

At $\mathrm{BMC}$, research is always in progress.

Learn more biomedcentral.com/submissions 